\title{
Tecnología, geoposicionamiento y cultura digital: navegando hacia la coproducción de la innovación científica
}

\author{
Elvira Santiago Gómez* \\ Kenedy Pedro Alva Chavez** \\ Sara Degli Esposti***
}

Recibido el 18 de marzo de 2016; aceptado el 20 de septiembre de 2016

\begin{abstract}
The Information and Communication Technology (ICT) revolution is transforming all spheres of social reality, leading many observers to talk about the emergence of a new digital culture. Sustained by the promises of the creation of a more solidarious, open and democratic culture, the information society is nowadays a reality, which permeates any sort of human expression. The widespread use of smartphones and geolocation systems (GPS) is a clear example of the transformative power of ICT. The extensive implementation of GPS in mobile devices has produced a significant growth in the supply of location-based services, usually available in the form of "apps" which offer great advantages to their users. Nevertheless their use entails high risks to users' information security and privacy: besides the risk of cybercrime, users of ICT services are not prepared to understand the complexity of the technology and the technical characteristics of most service settings. In order to use novel digital systems, unaware users often take uninformed decisions, facing the risk of losing part of their security, privacy, or of other fundamental rights related to freedom of movement and to one's ability to remain unseen. For this reason, it becomes extremely important to investigate the controversial nature of applications based on geolocation systems from a sociological perspective. Without any intention to stop, or prevent, technological progress, or to jeopardize the benefit the

* Universidad de Coruña (UDC), España, correo electrónico: elvira.santiago@udc.es

** Universidad de Coruña (UDC), España, correo electrónico: kalva@ucm.es

*** Asociación Española para el Fomento de la Seguridad de la Información (ISMS), España, correo electrónico: sara.degliesposti@ismsforum.es
\end{abstract}


industry is producing, this paper proposes a theoretical and empirical framework that aims at ensuring that all stakeholders will have a say and be represented in the innovation process. More specifically, we consider that responsible innovative technologies can only be the result of the collaboration and participation of all actors responsible for the advancement of ICT systems. These advancements are based on a shared digital ethics that ensures the protection of both the security and privacy of users. For this purpose, we analyse the results of a Citizen Summit conducted in Spain in 2014. During this event, Spanish citizens had the chance to discuss their priorities and to give recommendations on the production of geolocation applications, formulating shared principles that can guide the development of geolocalisation-based apps proceeding from a real responsible innovation process.

Key words: Geolocation, Information and Comunication Technologies, Coproduction, Ethics, Participation.

\section{Resumo}

A revolução das Tecnologias da Informação e de Comunicação (TIC) supõe a transformação de todas as esferas da realidade social e anima os autores a falar da emergência de uma nova cultura digital também conhecida como sociedade da informação, sociedade em rede ou sociedade líquida. Amparada nas promessas de uma construção social mais solidária, aberta e democrática, a sociedade da informação é hoje em dia uma realidade que tem impregnado os esforços sociais de realização humana. Um claro exemplo do poder transformador das novas TIC é o que se desprende do uso generalizado dos smartphones com sistemas de geolocalização integrado (GPS). A implantação massiva de GPS nos dispositivos de telefonia móvel tem levado a um crescimento na prestação de serviços baseados em endereços que normalmente estão disponíveis em forma de aplicações, apps, oferecendo a seus usuários grandes facilidades e serviços relacionados com a mobilidade. Apesar das vantagens, a utilização destas aplicações baseadas em endereços supõe também elevados riscos para a segurança e a privacidade, os usuários das aplicações baseadas em geoposicionamento se expõem a especificidades tecnológicas complexas relacionadas com os ajustes técnicos dos serviços ofertados, para poder utilizar as novas TIC, os usuários a princípio tomam decisões desinformadas em função do risco de sacrificar parte de sua segurança de forma pouco pensada e colocando em perigo sua verdadeira libertade de movimento. Ante esta complexa realidade, a tese central deste artigo sustenta que as aplicações baseadas na geolocalização se convertem em um objeto plano, em termos latourianos, um objeto relevante para seu estudo sociológico desde uma perspectiva renovada que respeita as premissas da co-produção da inovação tecnológica responsável. Com este objeto se propõe um enfoque teórico-empírico em que se garanta que todos os atores se encontrem representados e incluídos no processo de 
inovação das novas TIC baseadas em geoposicionamento. Através da participação e da deliberação de todos os atores sociais se poderá construir um cenário de progresso tecnológico baseado na confiança e na ética digital compartilhada que garanta a proteção da segurança e da privacidade dos cidadãos e permita um entorno de utilização ético, seguro e livre de abusos. Seguindo este desejo se apresentam os resultados de análises da Conferência Cidadã organizada na Espanha em Junho de 2014 quando cidadãos tiveram a oportunidade de debater e formular uma série de princípios divididos que poderão guiar o desenvolvimento da primera geração de aplicações baseadas em geoposicionamento que respeite um processo de inovação responsável.

Palavras chave: Geoposicionamento, Tecnologias da Informação e da Comunicação, Co-produção, Ética, Participação.

\section{Resumen}

La revolución de las Tecnologías de la Información y de la Comunicación (TIC) supone la transformación de todas las esferas de la realidad social y anima a los autores a hablar de la emergencia de una nueva cultura digital también conocida como sociedad de la información, sociedad red o sociedad líquida. Amparada en las promesas de una construcción social más solidaria, abierta y democrática, la sociedad de la información es hoy en día una realidad que ha impregnado los esfuerzos sociales de realización humana. Un claro ejemplo del poder transformador de las nuevas TIC es el que se desprende del uso generalizado de los smartphones con sistemas de geolocalización integrado (GPS). La implantación masiva de GPS en los dispositivos de telefonía móvil ha conllevado un crecimiento en la prestación de servicios basados en la ubicación que normalmente están disponibles en forma de aplicaciones, apps, ofreciendo a sus usuarios grandes facilidades y servicios relacionados con la movilidad. A pesar de sus ventajas, la utilización de estas aplicaciones basadas en la ubicación supone también elevados riesgos para la seguridad y la privacidad, los usuarios de las aplicaciones basadas en geoposicionamiento se exponen a especificidades tecnológicas complejas relacionadas con los ajustes técnicos de los servicios ofertados, para poder utilizar las nuevas TIC, los usuarios a menudo toman decisiones desinformadas bajo el riesgo de sacrificar parte de su seguridad de forma poco reflexionada y poniendo en peligro su verdadera libertad de movimiento. Ante esta compleja realidad, la tesis central de este artículo sostiene que las aplicaciones basadas en la geolocalización se convierten en un objeto peludo, en términos latourianos, un objeto relevante para su estudio sociológico desde una perspectiva renovada que respete las premisas de la coproducción de la innovación tecnológica responsable. Con este objeto se propone un enfoque teórico-empírico en el que se garantice que todos los actores se encuentran representados e incluidos en el proceso de innovación de las nuevas TIC 
basadas en geoposicionamiento. A través de la participación y la deliberación de todos los actores sociales se podrá construir un escenario de progreso tecnológico basado en la confianza y en la ética digital compartida que garantice la protección de la seguridad y la privacidad de los ciudadanos y permita un entorno de utilización ético, seguro y libre de abusos. Siguiendo este anhelo se presentan los resultados del análisis de la conferencia ciudadana organizada en España en junio de 2014 en la que los ciudadanos tuvieron la oportunidad de debatir y formular una serie de principios compartidos que podrán guiar el desarrollo de la primera generación de aplicaciones basadas en geoposicionamiento que respete un proceso de innovación responsable.

Palabras clave: geoposicionamiento, Tecnologías de la Información y la Comunicación, coproducción, ética, participación.

\section{Introducción}

Durante sus 40 años de existencia, Internet se ha convertido en una herramienta cada día más poderosa e intuitiva, capaz de dar respuesta a las necesidades del hogar y del trabajo. Algunos pensadores como Baudrillard (1981) sostienen una visión pesimista ante el poder transformador de las nuevas Tecnologías de la Información y la Comunicación (TIC) y advierten que nos encontramos ante una amenaza apocalíptica de des-realización generalizada o desaparición universal del mudo tal y como lo conocíamos; otros como Lévi (1999), adoptan posiciones más optimistas que abogan por la evolución de la hominización a través de la virtualización. Nuestra posición supera esta dicotomía y se enfrenta a la controversia que supone el poder transformador de las TIC desde una mirada sociológica ampliada en la que los ciudadanos se reconocen como participantes activos en la sociedad del conocimiento en tanto que consumidores y productores de contenidos digitales. Entre los desafíos de esta nueva realidad está el alcanzar una ética digital compartida que supere las barreras nacionales y oriente el desarrollo y la implantación de las nuevas TIC basadas en geoposicionamiento bajo unas premisas que garanticen un proceso de innovación responsable. Con esta propuesta se persigue que los ciudadanos dejen de ser considerados meros consumidores de contenidos digitales y se conviertan en un actor relevante en el proceso de innovación digital tomando como caso de estudio el desarrollo de nuevas tecnologías basadas en la geolocalización.

A tal fin, el contenido de este artículo revisa el contexto internacional de crecimiento y universalización en la accesibilidad de las TIC y de las aplicaciones basadas en geoposicionamiento; repasa las corrientes teóricas desde las que se defiende la coproducción del avance científico-técnico a través de la implicación temprana de todos los actores garantizando procesos de innovación responsable; y se presenta el análisis empírico de la reunión ciudadana mantenida en España en junio de 2014, como parte de las actividades de investigación programadas por el Proyecto 
Surprise $^{1}$ (Surveillance, Privacy and Security: a large scale participatory assessment of criteria and factors determining acceptability and acceptance of security technologies in Europe) financiado por la Comisión Europea de Investigación bajo su Séptimo Programa Marco.

\section{La universalización de la cultura digital y el poder transformador de las TIC}

Según datos del Banco Mundial y de la Unión Internacional de las Telecomunicaciones, el porcentaje de individuos que utilizan Internet a lo largo del planeta no ha dejado de crecer en los últimos años. Como se puede observar en la evolución que muestra el mapa de la Figura 1, en la última década Internet ha dejado de ser una herramienta utilizada mayoritariamente en Europa y Estados Unidos para convertirse en una realidad global a la que muy pocos países escapan. Y es que amparada en las promesas de una construcción social más solidaria, abierta y democrática, la sociedad de la información es hoy en día una realidad que ha impregnado los esfuerzos sociales de realización humana (Mattelart, 2002).

Ya nadie duda hoy en día del enorme potencial y beneficio — ni tampoco del impacto - que las tecnologías digitales suponen en nuestra vida. La cultura digital se expresa y se materializa en los medios digitales o electrónicos que se han arraigado en la vida cotidiana (Revés y Nass, 1996; Paper, Holmes y Popovich, 2004; Leivnouw y Livingstone, 2002; Deuze, 2006). El enorme potencial transformador de las TIC se recoge en la Agenda Digital Europea en la que se destaca su capacidad para reducir el consumo de energía, perfeccionar los servicios de salud u ofrecer mejores servicios públicos. Los nuevos artefactos extienden nuestra capacidad de comunicarnos apreciándose una nueva relación entre humano y máquina (Wellman, 2002; Castells, 2005). La nueva cultura digital se caracteriza entonces por la dificultad de discernir los artefactos, de las actividades y de los humanos creándose nuevas identidades digitales (Latour, 2005). Así, los principales componentes de esta cultura digital se encuentran en los valores y las prácticas que la gente emplea para tener la "libertad" relativa de hacer y tener una identidad digital (Bauman, 2004) con la que participar en la política de la vida (Giddens, 1991). Si bien la mera existencia de lo que denominamos una cultura digital no implica necesariamente que todos los individuos interactúen y se relacionen en el mundo online, dado el grado de penetración de las TIC resulta difícil que puedan existir personas que no siendo usuarios directos de estas tecnologías no se vean afectados de forma directa o indirecta en su forma de vivir y de relacionarse por esta nueva realidad.

Sin embargo, pese a las grandes oportunidades y ventajas que ofrecen las TIC, sus bondades se ven en ocasiones ensombrecidas por los malos usos, y es que los

\footnotetext{
La información detallada sobre el proyecto y los resultados puede consultarse en <www.surpriseproject.eu>.
} 
incidentes de seguridad cibernética están aumentando en frecuencia y magnitud hasta el punto de situarse en uno de los tres ejes de actuación de la Agenda de Seguridad Europea (EC, 2015). Hasta la fecha, las estadísticas y los informes publicados, tanto por organismos oficiales como por medios de comunicación o empresas especializadas, arrojan cifras de crecimiento económico, penetración, actividades y cambio de valores como resultado de la utilización e implantación de las TIC, a título de ejemplo y según recoge el Eurobarómetro 423 sobre CiberSeguridad publicado en noviembre de 2014 por la Comisión Europea, el 85\% de los españoles utilizan ya su smartphone para acceder a Internet, dato que sitúa a España veinte puntos por encima de la media europea. Pero, parejo al despliegue de aplicaciones y de nuevos usos de Internet crecen también las preocupaciones ligadas a su utilización relacionadas con la protección de la privacidad, la confianza en las instituciones encargadas de la gestión de los datos o la preocupación por el fraude. Y es que en los últimos años y siempre sobre la premisa de la funcionalidad de las TIC parece que se está produciendo un matrimonio entre un estilo de vida tutelado por la primacía de un valor científico-industrial y social por sobre un derecho natural a una vida privada (Matterlart, 2002). Estas nuevas amenazas a las que se enfrenta el mundo de las TIC se reflejan también en las actitudes de los ciudadanos hacia la actividad online como puede observarse en los resultados del Barómetro de mayo de 2013 publicado por el Centro de Investigaciones Sociológicas que muestra que el $76.3 \%$ de los entrevistados se encuentran preocupados por la protección de datos personales y el posible uso fraudulento de información personal que puedan realizar terceros.

Un claro ejemplo del poder transformador de las TIC en nuestra sociedad y nuestra cultura es el que se desprende del uso generalizado de la telefonía móvil y en concreto de los smartphones y los sistemas de geolocalización que a menudo les son inherentes. Durante estos años el avance de la tecnología inalámbrica ha permitido que los usuarios puedan navegar desde sus dispositivos móviles de forma estática y dinámica acercando más si cabe a los ciudadanos de los distintos países al uso y las posibilidades que Internet les ofrece con independencia, en muchas ocasiones, del grado de desarrollo económico e industrial del país. Si observamos el mapa de la Figura 2 podemos ver como la penetración de los dispositivos móviles ha puesto por delante de Estados Unidos y Europa a Latinoamérica y el norte de África. En 2014, se contabilizaron en toda América Latina más de 270 millones de smartphones, un 54.6\% más que en 2013, según cifras de la compañía Ericsson. La penetración de la telefonía móvil en Latinoamérica ha alcanzado un $112 \%$, cuando en la media global es de $85 \%$ y en España alcanza el 108\%. Si en una etapa inicial el desarrollo de la telefonía móvil nace como un mecanismo de comunicación que permite una mayor flexibilidad espacial y libertad de movimiento de los usuarios de telefonía que ya no se ven restringidos al radio espacial que abarca el cable que los 
conectaba a la red, hoy en día los usuarios de smartphones, pese a tener la posibilidad de comunicarse en un radio que abarca la casi totalidad del planeta, están siempre y en todo momento geolocalizados, produciéndose una particular paradoja entre la libertad de movimiento que los nuevos smartphone permiten y la continua vigilancia a la que se somete a los usuarios.

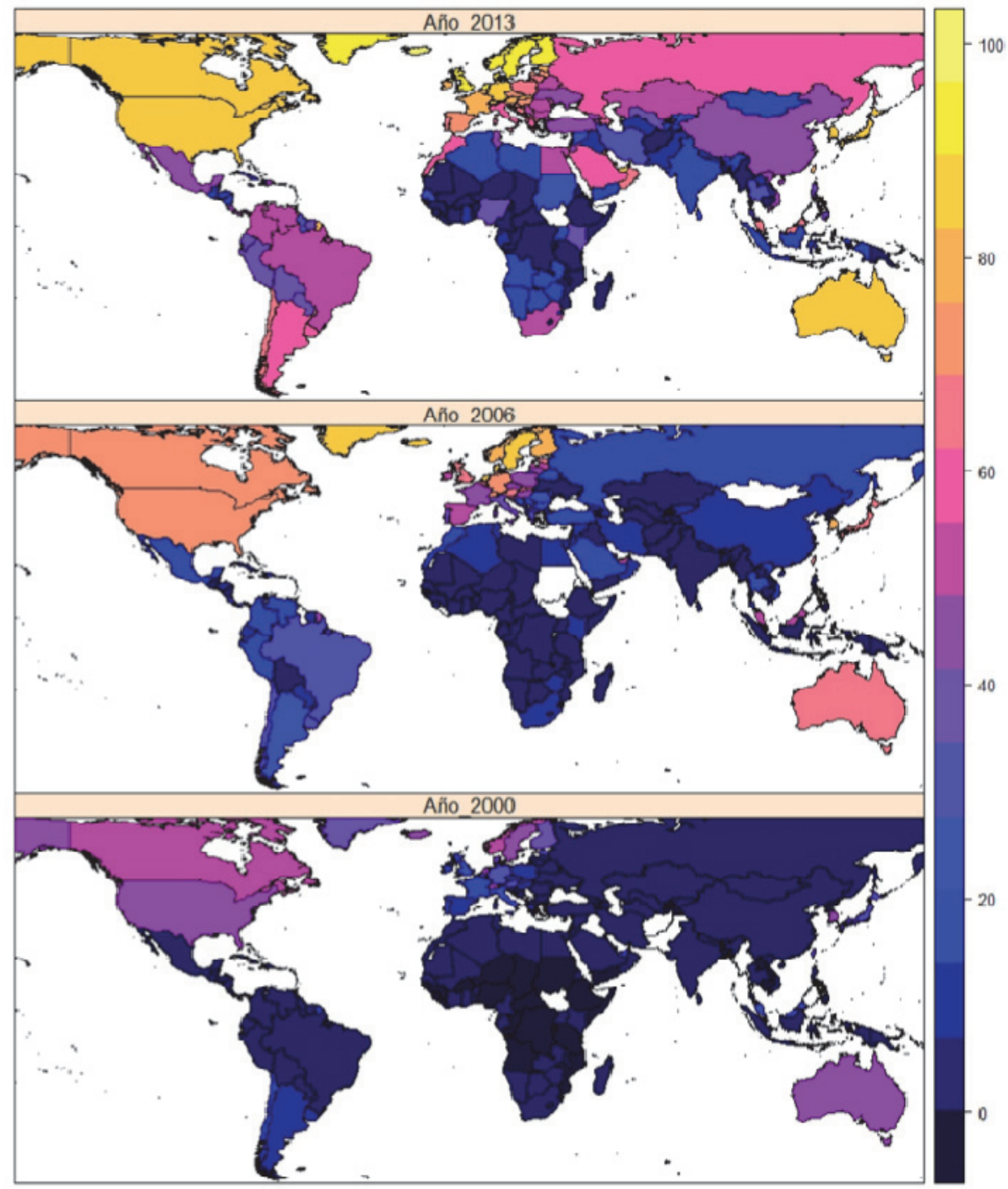

Figura 1. Evolución del acceso a Internet: porcentaje de individuos que utilizan Internet. Fuente: Banco Mundial. Elaboración propia. 


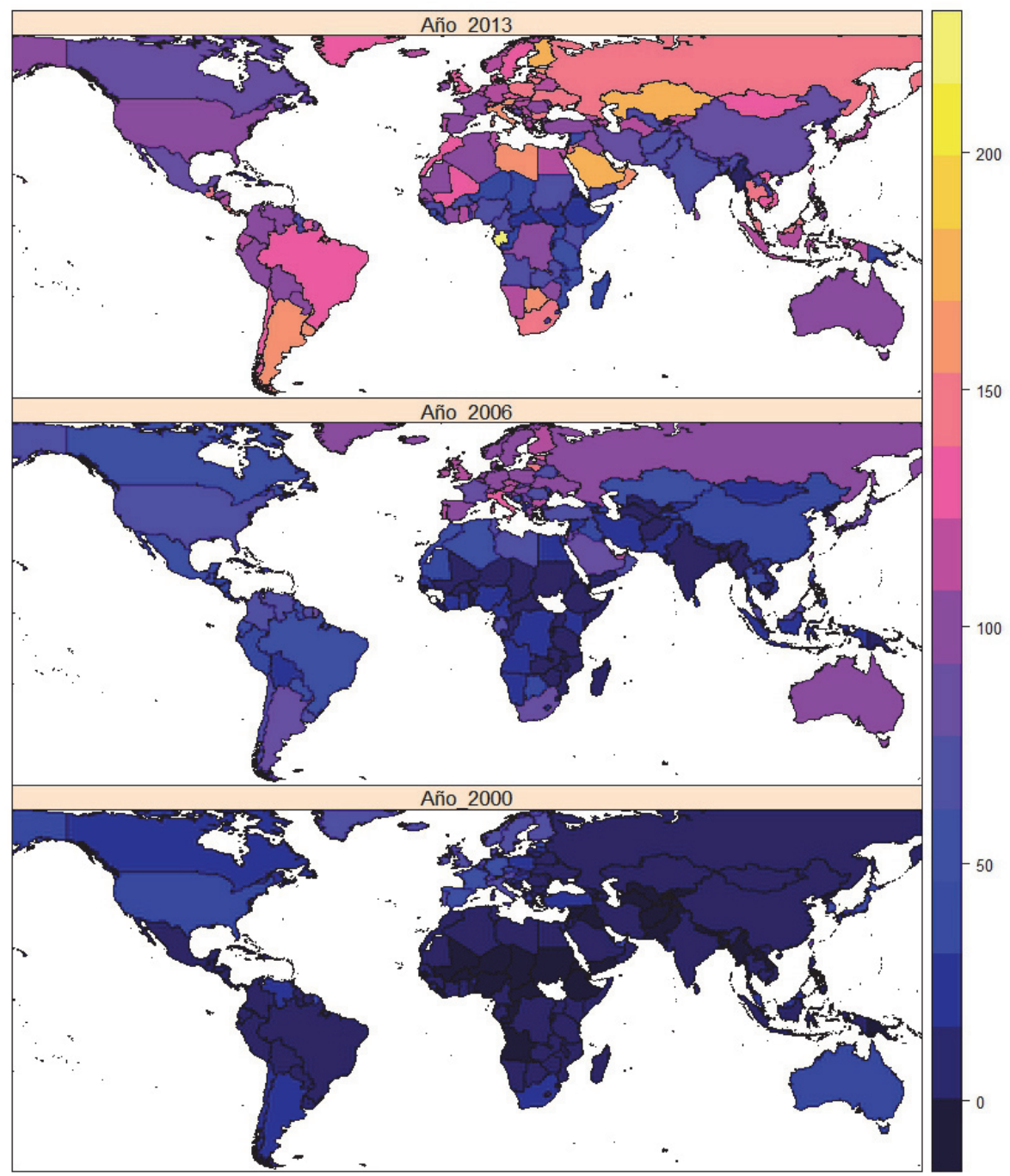

Figura 2. Evolución del acceso a indispositivos móvil: porcentaje de dispositivos móviles por cada 100 habitantes.

Fuente: Banco Mundial. Elaboración propia.

Las antenas de telefonía desempeñan un papel crucial para la localización de los teléfonos móviles, para poder conectarse a la red, realizar llamadas y enviar mensajes, todos los teléfonos móviles deben registrarse en la antena de telefonía más cercana que guarda siempre la ubicación del teléfono. Además la persona que utili- 
za el teléfono puede configurarlo de manera que determine su ubicación mediante satélites de posicionamiento global (GPS) o conectándose a redes inalámbricas.

Los defensores de la privacidad argumentan que el nivel de información que se puede obtener mediante la localización y seguimiento de un smartphone es preocupante y amenaza algunos de nuestros derechos fundamentales. A título de ejemplo, a raíz de las protestas del movimiento "Occupacy" en Nueva York, Twitter se vio obligada a facilitar datos de ubicación al gobierno estadounidense para identificar a los manifestantes, como reacción, se lanzó un nuevo servicio llamado "Please Don't Stalk Me" (por favor no me sigas), permitiendo a los usuarios ocultar los datos de ubicación asociados a sus tweets. Parejo a la pérdida de privacidad que la geolocalización supone y las cuestiones éticas al respecto, el seguimiento de nuestros pasos a través de la geolocalización tiene también sus ventajas comerciales, y es que las compañías utilizan los datos para diseñar tarifas de uso de servicios adaptadas a las necesidades de sus clientes. En definitiva, los usuarios no tienen un control total sobre la información que transmiten sus smartphones. Esto es especialmente delicado en el caso de los usuarios más vulnerables, como testigos protegidos, que pueden no querer compartir los datos sobre su ubicación, pero que necesitan un teléfono móvil.

\section{La coproducción de información geoespacial y el aumento de la participación ciudadana en los procesos de innovación tecnológica socialmente responsable}

El objetivo de alcanzar un proceso de innovación responsable respaldado en una ética digital compartida que recoja y respete las inquietudes de los ciudadanos en el desarrollo de aplicaciones basadas en geoposiciomamiento no resulta tarea fácil. El panorama mundial en el suministro y utilización de datos geoespaciales está cambiando muy rápidamente gracias a los avances que suponen los nuevos satélites y los dispositivos móviles lo que nos conduce a una reconfiguración de la relación tradicional entre la oferta y la demanda de este tipo de servicios así como de las relaciones entre un número creciente de actores involucrados en el proceso de innovación (Craglia y Shanley, 2015). Las oportunidades que las aplicaciones de la información geoespacial puedan ofrecer en el futuro parecen no tener límites una vez se implante el Internet de las Cosas (Internet of things). Que las nuevas aplicaciones resulten beneficiosos o perjudiciales para el conjunto de la sociedad dependerá de si el proceso de innovación se sustenta en una ética compartida y respete las pautas de la innovación y la investigación responsable.

La naturaleza controvertida de las aplicaciones basadas en la geolocalización, hace de éstas un objeto relevante para su estudio sociológico y es que se presenta al mismo tiempo como una nueva tecnología llena de posibilidades y con un marcado carácter innovador; pero al mismo tiempo, su utilización supone elevados riesgos para la privacidad de sus usuarios y pone en peligro los derechos fundamentales de 
nuestra cultura relacionados con la libertad de movimientos y la privacidad. A la hora de enfrentarnos como sociedad a esta nueva realidad resulta conveniente hacerlo desde un aproximación teórico-empírica en la que se garantice que todos los actores se encuentran representados reduciéndose las posibles hostilidades o el rechazo de los productos finales. Algunas de las preguntas que se plantean los teóricos de la coproducción de la innovación tecnológica se refieren a los riesgos a los que se enfrenta este nuevo esquema de producción, su sostenibilidad en términos económicos y la calidad de los inputs.

Seamos capaces o no de responder a estas preguntas, confiar en la democratización de la producción del conocimiento científico y del avance de la tecnología nos conduce en todo caso a re-examinar el contrato tradicional establecido entre productores de tecnología y consumidores. La noción de sociedad del Modo 2 que presentan Helga Nowotny, Peter Scott y Michael Gibbons (2001) se construye sobre la idea de que el conocimiento científico y tecnológico no es un conocimiento exacto, sino que se trata de un conocimiento limitado e incierto. Además, muchas veces, la investigación no está orientada a servir las necesidades sociales. En otras palabras, las soluciones técnicas deberían ser generadas desde los contextos político-sociales particulares y la investigación debería estar orientada hacia fines aplicados concretos, o lo que inglés se conoce como goal driven research (Gibbons, Nowotny et al., 1994, 2001). Los participantes en la ciencia se han vuelto más conscientes de la manera en que la ciencia y la tecnología afectan a los intereses y valores de la sociedad y, por lo tanto, se debe investigar atendiendo a la contextualización de los discursos de los diferentes stakeholders lo que permitirá alcanzar un conocimiento social robusto y una ciencia socialmente más responsable.

Si bien la noción de producción de conocimiento del Modo 2 ha suscitado un interés considerable, no se trata de una propuesta universalmente aceptada y entre sus críticos se señalan tres tipos de problemas que afectan a su validez empírica, su fuerza conceptual y su valor político (Hessels y Van Lente, 2008). En cuanto a la validez empírica, Etzkowitz y Leydesdorff (2000) sostienen que el Modo 2 no es una propuesta nueva, sino que resulta el formato original de la ciencia previa a su institucionalización académica en el siglo XX. En cuanto a la fuerza conceptual del Modo 2, se ha argumentado que la coherencia de sus funciones es cuestionable, en el sentido de que la multidisciplinariedad llevada al extremo puede afectar al control de la calidad de los resultados de la actividad científica (Rip, 2002). Por último, se critica la forma en que Gibbons y sus colaboradores mezclan elementos descriptivos y normativos, según Godin (1998) el Modo 2 es más una ideología política que una teoría descriptiva.

De alguna manera complementaria del enfoque anterior, pero con un semblante más crítico, aparece el enfoque de la coproducción de la ciencia, inspirado en los escritos de Jasanoff $(1996,2004)$. Su modelo analítico se encuentra apoyado por 
autores como Felt (2007) y su objetivo principal trata de proporcionar una perspectiva teórica sobre la mutua constitución del orden social y la tecnociencia. En el enfoque de la coproducción de la ciencia, los tres ámbitos, ciencia, tecnología y sociedad son el resultado de un proceso en que los actores políticos, científicos y sociales interactúan con el objetivo de determinar las direcciones y las prioridades de los avances y actividades científicas. No se trata tanto de la creación de nuevos mecanismos participativos como de establecer nuevas formas de producción de conocimiento. Sheila Jassanoff $(1996,2000,2004)$, ha afirmado de manera convincente "la ciencia y la innovación tecnológica no se pueden enfocar como actividades neutrales, cualitativamente distinta de otras actividades sociales, ya que la ciencia y la tecnología son el resultado de un proceso de coproducción en el que los agentes sociales, políticos y científicos interactúan y al final determinan las direcciones, las prioridades y los avances de la actividad científica".

Los autores que defienden la coproducción proponen que se establezca un compromiso público con la ciencia en todo el proceso de producción y que no se limite la participación pública a las etapas del proceso científico como suele suceder en la práctica y a través de los procesos de evaluación y validación de las nuevas tecnologías (technolgy assessment) (Wilsdon y Willis, 2004). Si la ciencia es por definición una cuestión de coproducción, entonces es posible preguntarse si este proceso está limitado a algunos tipos de actores o si todas las partes deberían tener un acceso y una influencia equitativa; en el contexto de los procesos de ciencia e innovación de la UE, se ha observado a menudo que los conocimientos técnicos, en alianza con la industria, han llegado a dominar las estrategias de coproducción (Ferretti y Pavone, 2009). Pese al esfuerzo realizado por poner en marcha procesos de coproducción, resulta necesario ampliar la perspectiva participativa capacitando al conjunto de los ciudadanos — sean detractores o defensores de la tecnología en cuestión- e incluirlos en el proceso innovativo como actores fundamentales en el proceso de creación, producción de conocimiento y avance tecnológico. Para conseguir que investigadores, ciudadanos, políticos, empresas, organizaciones del tercer sector, y el resto de posibles actores, trabajen juntos durante todo el proceso de investigación e innovación, con el objetivo último de que los resultados alcanzados coincidan con los valores, necesidades y expectativas de la sociedad europea, en el contexto de la estrategia europea de investigación H2020 se define la "Investigación e Innovación Responsable" (RRI, por sus siglas en inglés). El término ha adquirido cada vez mayor relevancia política, en particular dentro del programa "Ciencia por y para la Sociedad" y define la investigación y la innovación responsable como aquella en la que se garantiza: a) la participación ciudadana en la ciencia, de manera que los ciudadanos desarrollen intereses y capacidades hacia ella, que les permitan participar activamente en actividades científicas; b) en el que se garantice el compromiso de los investigadores con la sociedad, haciendo más visi- 
bles las dimensiones sociales y los objetivos del esfuerzo científico; c) el aumento de la importancia de las políticas de investigación e innovación para la sociedad, que permita a los responsables políticos y organismos financiadores adaptarse a las condiciones para inducir una investigación e innovación cercana a la sociedad. ${ }^{2}$

En el caso concreto de las aplicaciones basadas en geoposicionamiento, en un intento de clasificar la pluralidad de mecanismos de coproducción en este campo, Craglia y Shanley (2015) establecen la siguiente categorización. En primer lugar estarían los proyectos de ciencia ciudadana, en los que se incluyen aquellas experiencias en las que el público participa en auténticas investigaciones científicas. La Ciencia Ciudadana se establece como una forma abierta de colaboración entre miembros de la sociedad que participan en el proceso científico, bien planteando preguntas, recogiendo o procesando información o interpretando los resultados (Bonney, 2014). Uno de los elementos comunes de los proyectos de ciencia ciudadana es que implican una fuerte interacción entre la comunidad académica y la esfera pública, pero la metodología de los proyectos por lo general está diseñada por los investigadores con un bajo grado de implicación de los participantes de la esfera pública (Shirk et al., 2012; Haklay, 2011; Newman et al., 2012). En segundo lugar se encontrarían los proyectos de crowdsourcing, e incluye aquellas iniciativas en las que los individuos u organizaciones solicitan la contribución de un elevado grupo de individuos desconocidos en algunos casos o de un grupo acotado de personas o expertos de confianza (Bowser y Shanley 2013, 45). En la mayoría de estos proyectos, la metodología para la recolección y análisis se diseña también de forma centralizada por los investigadores. En tercer lugar estarían la generación de contenido ciudadano o el data mining. Esta categoría de proyectos difiere de los anteriores en que no producen información primaria sino que se basan en la reutilización de publicaciones y contenidos ya disponibles en Internet que se reutiliza para otros fines diferentes de su objetivo inicial, los ejemplos incluyen la detección de incendios forestales a través de las publicaciones en Twitter (Craglia, Osterman y Spinsanti, 2012), o la cartografía de crisis (Shanley et al., 2013 ).

Aplicar las premisas de la Investigación e Innovación Responsable al campo de las nuevas tecnologías de la información y la comunicación para que los productos que resulten sean socialmente aceptados y respondan a los valores y necesidades de la sociedad resulta especialmente controvertido dado el elevado grado de intrusividad en la vida privada que la utilización de las nuevas TIC puede suponer. En la recopilación de Schomberg (2011) se advierte de la necesidad de alinear la innovación con las necesidades y valores de la sociedad a través de la participación temprana de los distintos actores y grupos de interés, que supere las limitaciones de las prácticas tradicionales de evaluación de tecnologías. Entre los métodos que Von

Véase $<$ http://ec.europa.eu/programmes/horizon2020/en/h2020-section/responsible-research-innovation>. 
Schomberg señala como adecuados para alcanzar una innovación responsable de las tecnologías de información y comunicación. En la misma línea, la innovación responsable en el campo de las nuevas tecnologías de la información y la comunicación debe ir acompañada de la creación de plataformas de deliberación e inclusión de los ciudadanos y de la sociedad civil.

\section{Apunte metodológico}

La metodología empleada en España, dentro del marco de actividades planteadas por el proyecto Surprise, para la identificación de las prioridades ciudadanas y eventuales conflictos éticos en el proceso de innovación responsable de aplicaciones basadas en geoposicionamiento confía en las ventajas del diálogo abierto entre ciudadanos, políticos y expertos, en el que los actores co-producirán una definición deliberada de los problemas y conflictos que supone la innovación en tecnologías de geoposicionamiento y colaborarán en la búsqueda y en el diseño de un marco regulador que garantice no solo que el producto final será socialmente aceptado sino también su utilización; y en el compromiso fundamentado en acciones de participación ciudadana cuya premisa principal es el intercambio de conocimiento y experiencia en una doble dirección: desde los expertos y responsables políticos hacia los ciudadanos; y desde los ciudadanos a expertos y responsables políticos.

En las reuniones ciudadanas organizadas en el año 2014 bajo el marco de actividades del proyecto Surprise, el objetivo era identificar los factores y criterios de aceptabilidad social de distintas tecnologías que pueden emplearse con fines de seguridad, entre ellas, las basadas en la geolocalización mediante smartphones. Antes de participar en la reunión cada ciudadano recibió en su casa una revista en la que se le informaba de los usos, ventajas e inconvenientes que un grupo de expertos formado por representantes de la industria, la política, grupos de usuarios y comunidad científica habían previamente identificado con respecto al uso de cada una de las tecnologías de seguridad seleccionadas: drones, cámaras de videovigilanca, geolocalización mediante smartphones, inspección profunda de paquetes y biometría. La misma información podía consultarse en los videos informativos producidos para apoyar el contenido de las revistas y avivar el debate ${ }^{3}$ en las mesas. En la primera reunión, celebrada en febrero de 2014, participaron 250 ciudadanos repartidos en 30 mesas de deliberación, y en la segunda reunión el número de participantes se redujo a 35 repartidos en cinco mesas de deliberación, en la que los ciudadanos debatieron sobre las prioridades que para ellos deben respetarse en el proceso de innovación tecnológica, ofreciendo recomendaciones a industria y policy-makers. La técnica del grupo de discusión empleada en las mesas está diseñada para permi-

\footnotetext{
Los materiales informativos para apoyar la deliberación en las mesas pueden consultarse en $<$ www.surprise-project.eu $>$.
} 
tir la libre expresión de los participantes y que sea la propia discusión la que genere los temas a tratar. Esta apertura discursiva es la que se pretende en esta ocasión bajo la convicción de que la libre expresión de los hablantes hace aflorar en el discurso sus auténticas percepciones y preocupaciones. No obstante, en este caso se consideró oportuno que los participantes contasen con la opinión de los otros grupos o actores que forman parte del proceso de innovación tecnológica a través del material recogido en los videos y en las revistas informativas (Conde, 2012).

En este artículo se ofrece el resultado del análisis cualitativo de los repertorios discursivos y las configuraciones narrativas sobre las aplicaciones basadas en geoposicionamiento. El análisis trata de ofrecer un hilo conductor consistente desde el que se comprende el discurso de la población sobre el proceso de innovación de aplicaciones basadas en geoposicionamiento y se conecta el sentido general del debate concreto mantenido en cada uno de los grupos con el contexto global de innovación. Las perspectivas grupales dominantes en los grupos configuran las principales líneas de polarización y construcción de los discursos sociales, de ahí la importancia de su identificación y análisis ya que suministran los criterios de representación social; en este sentido los discursos producidos desde dicha posición en la micro-situación social del grupo se pueden considerar equivalentes y generalizables a los discursos producidos en los lugares sociales análogos que se ocupan en la vida real (Conde, 2012). La riqueza explicativa de las configuraciones narrativas y los espacios semánticos reside en la identificación de los discursos dominantes y de las principales tensiones o polarizaciones entre ellos. La identificación de la polarización de los discursos permite identificar las prioridades éticas a las que se debe atender en el diseño, fabricación y uso de aplicaciones basadas en geoposicionamiento para que se garantice la innovación responsable.

\section{La configuración narrativa sobre la innovación responsable de aplicaciones basadas en geoposicionamiento}

El discurso de los participantes en la reunión ciudadana sobre la innovación tecnológica relacionado con las aplicaciones basadas en geoposicionamiento se estructura a través de dos dimensiones principales que en la representación de la configuración narrativa se representan en el eje horizontal y vertical (véase Figura 3).

El primero de los ejes, el horizontal, es el eje de la valoración de la utilización de las aplicaciones basadas en geoposicionamiento, y en los extremos encontramos las ventajas y las desventajas que pueden desprenderse de su uso. Los participantes en la reunión ciudadana celebrada en España en junio de 2014 están ampliamente familiarizados con el uso de aplicaciones basadas en geoposicionamiento a través de sus propios smartphones. No obstante la valoración que realizan sobre su utilidad resulta ambivalente, y los ciudadanos consideran que las ventajas que presentan este tipo de aplicaciones se ven ensombrecidas por las desventajas; estar permanen- 
temente geolocalizado tendrá una valoración positiva o negativa en función de cada contexto y de cada momento. El segundo de los ejes, el vertical, es el eje de la percepción de la protección y en sus extremos se encuentran la sensación de seguridad y de inseguridad. La sensación de seguridad producida por la utilización de aplicaciones basadas en geoposicionamiento se percibe como una construcción social, algunos participantes, por ejemplo, sostienen que antes de la introducción de estos dispositivos la gente no relacionaba la protección y la seguridad personales con el seguimiento de la ubicación.

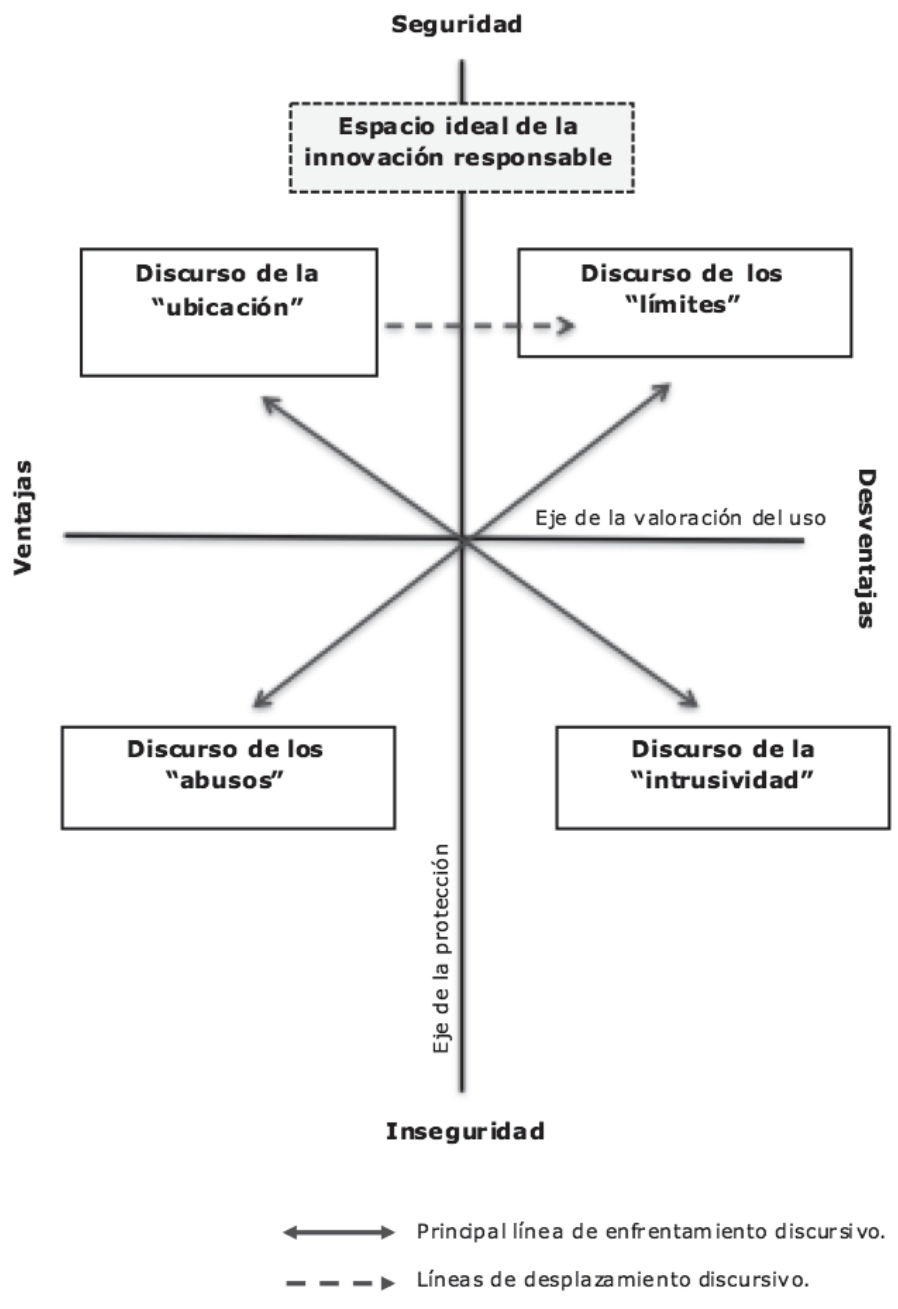

Figura 3. Configuración narrativa sobre la innovación responsable de aplicaciones basadas en geoposicionamiento. 
Del cruce de estas dos dimensiones se obtiene el espacio bidimensional sobre el que se asientan los cuatro discursos principales que conforman la configuración narrativa. En el cuadrante superior izquierda se encuentra el discurso sobre la ubicación. En este discurso se destacan las ventajas de la geolocalizacion y se reconoce la comodidad, la inmediatez, la efectividad y la rapidez de las aplicaciones y los servicios que permiten conocer tu propia ubicación o la de los lugares que necesitas geolocalizar. Las aplicaciones basadas en la ubicación mejoran la seguridad de los usuarios, bien sea por la sensación de confort que produce sentirse localizado, por la facilidad que supone la localización de un destino a través de un dispositivo GPS, o bien por la tranquilidad de saber que los equipos de emergencia pueden contar con este tipo de tecnología en caso de que necesidad (véase Extracto 1).

\section{Extracto 1. Discurso de la ubicación}

La principal ventaja es saber dónde estoy en todo momento, permite posicionar servicios negocios, y optimizar recursos a muy bajo coste como las rutas del tom tom (...) da efectividad porque cuando vas en coche pues lo miras en un momento y te va indicando, no tienes que pararte y mirar un mapa como hace mucho tiempo. Permite acercar el conocimiento del medio al ciudadano que es un poco conocer dónde estoy y con lo que me voy a encontrar con lo que voy a interaccionar, el medio natural (...) inmediatez para tú encontrar o para que te encuentren, para tener lo que necesitas en ese momento, que pueden ser cosas que tú necesitas o que lo que necesites sea que te tengan a ti localizado.

En el cuadrante inferior izquierda se asienta el discurso de los abusos. La población considera que hay que estar permanentemente en alerta para prevenir los abusos que pueda suponer la recopilación por parte de las empresas distribuidoras de servicios de la información sobre nuestra localización. Desde este discurso se destaca la autorresponsabilidad en la utilización de las tecnologías basadas en la ubicación como una de las claves que permitirían evitar que el usuario se convierta en víctima de un eventual abuso (véase Extracto 2).

\section{Extracto 2. Discurso sobre los abusos}

Ahora es verdad que cuando te instalas cualquier aplicación te dice cosas que tienes que aceptar, y tal y tal, entre ellas geolocalizarte, normalmente tú dices que sí, pero porque si no aceptas no funciona, peus podrían decirte, para qué quieren esos datos, y tú puedas valorar sí sí o si no (...) Las tecnologías que se dice que incrementan la seguridad necesitan en realidad que las personas se sientan inseguros para que puedan convertirse en herramientas rentables.

En el cuadrante inferior derecha se asienta el discurso sobre la intrusividad que este tipo de servicios suponen para nuestra vida privada. La población considera que las nuevas tecnologías basadas en la geolocalización son intrusivas para la 
privacidad, el principal beneficio de estos servicios, la propia capacidad de localización, se convierte de forma paradójica en su principal desventaja al coartar la libertad de movimientos. Desde este discurso se denuncia la recogida de información personal indiscriminada. La utilización de los datos personales sobre la geolocalización de los usuarios de aplicaciones móviles debería recogerse únicamente bajo una orden judicial y cuando se persigan unos objetivos claros y lícitos (véase Extracto 3).

\section{Extracto 3. Discurso sobre la intrusividad}

La geolocalización es lo que estamos hablando, te puede gustar o no pero yo creo que es lo más intrusivo que hay porque te está diciendo dónde estás en cada momento, si estás de acuerdo perfecto pero si no la ventaja de la localización se convierte en una desventaja que coarta tu libertad de movimientos (...) mis datos o los de cualquiera se deben manejar bajo una orden judicial y conforme a una serie de normas claras, te lo tienen que decir, te tienen que informar si no esto se convierte en un tribunal de la Santa Inquisición.

Por último, en el cuadrante superior derecha se encuentra el discurso de los límites. Desde este discurso los ciudadanos consideran que se debería elaborar un marco legislativo global que supere las barreras o las contradicciones que puedan existir entre la legislación del país que produce la tecnología y la legislación del país en la que ésta se utiliza; las TIC han conseguido una implantación globalizada que supera las fronteras nacionales, y el marco que las regula debe ser por tanto también un marco acordado a nivel internacional. Establecer límites a través de la regulación debe complementarse con la educación y concienciación a la ciudadanía en la importancia de prestar atención e involucrarse en el avance de la ciencia y la tecnología para que no se produzcan malos usos (véase Extracto 4).

\section{Extracto 4. Discurso sobre los límites}

Lo que verdaderamente me preocupa de la legislación, es que por ejemplo en Europa tenemos una ley más garantista que en EEUU, pero estamos utilizando muchas tecnologías de comunicación que son norteamericanas entonces son ellos, si el gobierno les pide a ellos que le den información se la van a dar, tal vez lo mejor sería que la ley que se aplique sea la del país del usuario o que los gobiernos se pongan de acuerdo para que podamos tener una legislación mundial para este tipo de cosas (...) tiene que haber una base de educación, una base de cultura para que la gente sea íntegra en sus trabajos y que la gente sepa responder y haya normas claras, pero si la persona está bien educada y concienciada tendrá mayor respeto por los demás.

Las principales polarizaciones en la configuración narrativa se producen entre el discurso de la ubicación y los discursos de los abusos y la intrusividad, estos dos últimos ensombrecen las ventajas y las mejoras para la seguridad que ofrecen los 
servicios basados en la geolocalización. Para combatir estos efectos negativos, la población considera que se debe trasladar el foco de atención hacia los límites y la regulación eficaz de este tipo de servicios. El poder transformador de las nuevas tecnologías debe limitarse a través de marcos normativos adecuados y mayor implicación de los actores sociales en el proceso de innovación responsable. Se trata de que sea la sociedad la que integre a la tecnología en lugar de que los ciudadanos deban adaptar su comportamiento para evitar los riesgos y abusos. Así, la tecnología habrá de circunscribirse a las normas y a las prioridades de la sociedad a la que pretende dar servicio.

\section{Conclusión}

La propia existencia o la mera aparición de una nueva aplicación tecnológica, transforma nuestro entorno y obliga a que la población modifique su comportamiento para adaptarse a sus exigencias y protegerse de los malos usos. Ante esta situación la innovación responsable de aplicaciones basadas en geoposicionamiento debe garantizar que las prioridades establecidas por los ciudadanos orienten el desarrollo de la tecnología y su implantación exitosa en el mercado.

A través de la apertura de espacios de deliberación los ciudadanos participan en el debate sobre la dirección que debe seguir el desarrollo científico técnico desde la fase inical de diseño de nuevas aplicaciones. En este sentido, los ciudadanos españoles que participaron en el proyecto Surprise establecieron las prioridades que deben respetarse en el desarrollo de aplicaciones basadas en geoposicionamiento, destacando la importancia de alcanzar un contexto normativo a nivel internacional que proteja a los ciudadanos — con independencia de su nacionalidad- de los posibles abusos que puedan producirse, garantizando la protección de la privacidad de la información y asegurando una total transparencia sobre los usos que puedan darse de la información personal recabada por este tipo de aplicaciones.

Los beneficios de la coproducción y de la innovación responsable de aplicaciones tecnológicas suponen una oportunidad de poner en marcha un nuevo diseño de generación de políticas públicas y de manejo de la innovación científico-técnica apoyado en la gobernanza deliberativa, que permita a los actores sociales, políticos y científicos colaborar en la identificación de las áreas prioritarias de investigación y avanzar en la búsqueda de soluciones compartidas a los posibles ámbitos controvertidos y en el diseño de aplicaciones basadas en el geoposicionamiento que resulten socialmente responsables. El ámbito de la gestión de la innovación científicotécnica, deja así de ser un campo limitado a legisladores, científicos e industria, como únicos colaboradores legítimos de los responsables políticos, avanzando en una gestión global y participativa que fomente la confianza en las instituciones y los lazos sociales entre actores lo que sin duda supondrá avanzar en la democracia 
dialógica y participativa; construyendo una nueva identidad acorde con la cultura digital en al que nos encontramos inmersos.

\section{Bibliografía}

Baudrillard, Jean, 1998 [1981]. "Simulacra and Simulations", Jean Baudrillard, Selected Writings, ed. Mark Poster, Palo Alto: Stanford University Press, pp. 166-184.

Bauman, Zygmunt (2004). Identity, Cambridge, Polity Press.

Bonney, R. (2014). "Citizen Science: A Vision for the Future", Keynote at the Citizen Cyberscience Summit, London, February 21.

Bowser, A. and L. Shanley (2013). "New Visions in Citizen Science", Woodrow Wilson Centre, <http:/www.wilsoncenter.org/publication/new-visions-citizenscience>.

Carolan, M. (2006). "Science, espertise and democratization of decision-making process", Society and Nature Resources, 19:661-668.

Castells, Manuel (ed.), 2005. The Network Society: A Cross-Cultural Perspective, Northampton, Edward Elgar Publishing.

Coleman, D.; Y. Georgiadou and J. Labonte (2009). "Volunteered Geographic Information: The Nature and Motivation of Producers", International Journal of Spatial Data Infrastructures Research, 4:332-358, <http://ijsdir.jrc.ec.europa. eu/index.php/ijsdir/article/view/140/223>.

Craglia, M.; F. Osterman and L. Spinsanti (2012). "Digital Earth from Vision to Practice: Making Sense of Citizen-Generated Content", International Journal of Digital Earth, 5:398-416.

Craglia, Max and Lea Shanley (2015). "Data democracy - increased supply of geospatial information and expanded participatory processes in the production of data", International Journal of Digital Earth, 8:9, 679-693.

Deuze, M. (2006). "Participation, remediation, bricolage: Considering principal components of a digital culture", The information society, 22(2), 63-75.

Etzkowitz, H. and Leydesdorff, L., (2000). "The dynamics of innovation: from National Systems and 'Mode 2' to a Triple Helix of university-industrygovernment relations", Research Policy, 29(2):109-123.

Felt, U. (coord.) (2007). Taking European knowledge society seriously, 6th Framework Programme, European Comission.

Ferretti, M.P. and Pavone, V. (2009). "What do civil society organisations expect from participation in science? Lessons from Germany and Spain on the issue of GMOs”, Science and Public Policy, 36(4):287-299.

Gibbons, M.; Camille, L. y Nowotny, H. et. al. (1994). The new Production of Knowledge: The Dynamics of Science and Research in Contemporary Societies, London, Sage. 
Giddens, Anthony (1991), Modernity and self-identity: self and society in the late modern age, Stanford, Stanford University Press.

Godin, B. (1998), "Writing performative history: the new new Atlantis?", Social Studies of Science, 28(3):465-483.

Haklay, M. (2010). "How Good Is Volunteered Geographical Information? A Comparative Study of OpenStreetMap and Ordnance Survey Datasets", en Environment and Planning B: Planning and Design 37:682-703.

(2011). "Citizen Science as Participatory Science", <http://povesham. wordpress.com/2011/11/27/citizen-science-as-participatory-science/>.

Hessels, K. y Van Lente, H. (2008). "Re-thinking new knowledge production: A literature review and researcha agenda", Research Policy, 37:740-760.

Jassanoff, S. (1996). "Beyond epistemology: relativism and egagement in the politics of science", Social Studies of Science, 26:393-418.

(2000). "Reconstructing the past, constructing the present: can science studies and the history of science live happily ever after?", Social Studies of Sicence, 30:621-31.

- (2004). "Science and citizenship: a new sinergy", Sciencie and Public policy, 31(2):90-94.

Latour, B. (2005). Reassembling the social. Politica y Sociedad.

Lévy, Pierre (1999). Cibercultura, Editora 34.

Lievrouw, Leah, and Sonia Livingstone (eds.) (2002). Handbook of New Media:

Social Shaping and Consequences of ICTs, London, Sage.

Matterlart, A. (2002). Historia de la sociedad de la información, Barcelona, Paidós.

Newman, G.; A. Wiggins; A. Crall; E. Graham; S. Newman and K. Crowston (2012). "The Future of Citizen Science: Emerging Technologies and Shifting Paradigms", Frontiers in Ecology and Environment, 10:298-304, $<$ http://www.esajournals.org/doi/abs/10.1890/110294>.

Nowotny, H. (2003). "Dilema of expertise", Science and Public Policy, 30(3):151156.

Nowotny, H. and Rose, H. (eds.) (1979). Counter-Movements in the Sciences. Sociology of the Sciences, vol. III, Reidel, Dordrecht.

Nowotny, H.; Scott, P. and Gibbons, M. (2001). Re-thinking Sciencie: Knowledge ant the Public in an Age of Uncertainty, Cambridge, Polity Press, pp.166-178.

Papper, Robert; Michael Holmes and Mark Popovich. 2004. "Middletown Media Studies", The International Digital Media \& Digital Arts Association Journal 1(1):1-56, <http://www.bsu.edu/icommunication/news/iDMAaJournal.pdf >.

Reeves, Byron and Clifford Nass (1996). The media equation: how people treat computers, televisions, and new media like real people and places, Stanford, CSLI Publications; New York, Cambridge University Press. 
Rip, A. (2002). "Science for the 21st Century", en Tindemans, Verrijin y Visser (eds.), The future of Science and the Humanities, Amsterdam, University Press, pp. 99-148.

Shanley, L.; R. Burns; Z. Bastian and E. Robson (2013) "Tweeting up a Storm", Photogrammetric Engineering \& Remote Sensing, October. <http://www.scribd. com/doc/174769846/Tweeting-Up-a-Storm-The-Promise-and-Perils-of-CrisisMapping>.

Von Schomberg, R. (2011). "Towards responsible research and innovation in the information and communication technologies and security technologies fields", Available at SSRN 2436399.

Wildson, J. y Willis, R. (2004). See-through science: why public engagement needs to move upstream, London, Demos.

Weingart, P. (1999). "Scientific expertise and political accountability: paradoxes of science in politics", Science and Public Policy, 26(3):151-161.

Wellman, Barry (2002). "Little Boxes, Glocalization, and Networked Individualism", Digital Cities II: Computational and Sociological Approaches (eds.) Makoto Tanabe, Peter Van den Besselaar and Toru Ishida, New York, Springer, pp. 10-25. 